Pathologe 2008 · [Suppl 2] 29:297-302 DOI 10.1007/s00292-008-1040-0

Online publiziert: 8

๑) Springer Medizin Verlag 2008

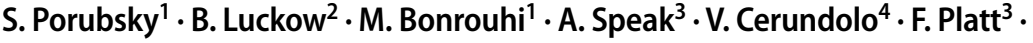
H.-J. Gröne ${ }^{1}$

${ }^{1}$ Zelluläre und molekulare Pathologie, Deutsches Krebsforschungszentrum, Heidelberg

${ }^{2}$ Medizinische Poliklinik, Universitätsklinikum München

${ }^{3}$ Department of Pharmacology, University of Oxford, UK

${ }^{4}$ Weatherall Institute of Molecular Medicine, University of Oxford, UK

\title{
Glykosphingolipide Gb3 und iGb3
}

\section{In-vivo-Rolle im hämolytisch-urämischen Syndrom und bei der Funktion der iNKT-Zellen}

Glykosphingolipide (GSL) sind amphipathische Moleküle, die aus einem sie in der Plasmamembran verankernden Ceramidanteil und aus einer unterschiedlich langen Zuckerrestkette bestehen. In Säugetieren leitet sich die Mehrheit der GSL vom Laktosylceramid (LacCer, d. h. GalGlcCer, Abb. 1) ab, von dem durch die Addition eines dritten Zuckerrestes bzw. eines Sulfats die einzelnen Gruppen der GSL entstehen (Abb. 1). Die Gruppe der Isogloboside unterscheidet sich von der Gruppe der Globoside lediglich durch die glykosidische Bindung zwischen dem 2 . und dem 3 . Zuckerrest ( $a 1-3$ bei Isoglobosiden und a1-4 bei Globosiden), der allerdings in beiden Fällen die Galaktose ist (Abb. 1). Die in vivo physiologische Rolle dieser 2 Gruppen der GSL bleibt weitgehend unbekannt.

Aufgrund von In-vitro-Versuchen wurde das Globotrihexosylceramid (Gb3, $\mathrm{CD} 77)$ als der Rezeptor für Verotoxin 1 und 2 impliziert $[4,9]$. Verotoxine sind Produkte enterohämorrhagischer Escherichiae coli (EHEC) und weisen eine hohe Homologie mit dem durch Shigella dysenteriae produzierten Toxin auf. Sowohl Vero- als auch Shiga-Toxine gehören wegen ihrer Struktur und ihrem Wirkmechanismus in die Gruppe der toxischen Lekti- ne wie z. B. auch Ricin. Verotoxine werden als das kausative Agens bei der Entstehung des hämolytisch-urämischen Syndroms und der thrombotisch-thrombozytopenischen Purpura angesehen. Sie bestehen aus einer enzymatisch aktiven A-Einheit und $5 \mathrm{~B}$-Einheiten, die für die Bindung an den membranständigen Zellrezeptor $\left(\mathrm{Gb}_{3}\right)$ verantwortlich sind. Nach der B-Einheit-vermittelten Bindung des Verotoxins an $\mathrm{Gb}_{3}$ erfolgt die Internalisation des Holotoxins mittels Endozytose, gefolgt von einem retrograden Transport ins endoplasmatische Retikulum [6, 15]. Die A-Einheit bewirkt dann dank ihrer N-Glykosidase-Aktivität die Spaltung der 28S-rRNA der 6oS-ribosomalen-Einheit und hemmt somit irreversibel die Proteinsynthese.

Unser Ziel war, in vivo zu testen, ob allein $\mathrm{Gb}_{3}$ (bzw. die Gruppe der durch die Gb3-Synthase synthetisierten GSL) für die Vermittlung der Verotoxintoxizität verantwortlich ist. $\mathrm{Zu}$ diesem Zwecke und zur Untersuchung weiterer In-vivo-Rollen der Globoside haben wir eine Gb3-Synthasedefiziente Maus generiert und die Toxizitätserscheinungen nach der Verabreichung von Verotoxin untersucht.

Isoglobotrihexosylceramid ( $\left.\mathrm{iGb}_{3}\right)$ hingegen, das sich nur durch eine glykosidische Bindung vom $\mathrm{Gb}_{3}$ unterscheidet, wurde als der endogene Ligand postuliert, der für die Entwicklung von inva- rianten „natural-killer-" T-Zellen (iNKT) verantwortlich ist. Natural-killer-T-Zellen (NKT) stellen eine diskrete Population von T-Zellen dar, welche neben dem T-Zell-Rezeptor (TCR) auch Marker der NK-Zellen (z. B. NK1.1) exprimieren [8]. Ein Unterteil der Population von NKT-Zellen, der als invariante NKT-Zellen (iNKT) bezeichnet wird, besitzt darüber hinaus einen TCR, welcher aus einer invarianten TCR- $\alpha$-Kette (Va14-Ja18 bei der Maus bzw. Va24-Ja18 beim Menschen) besteht. Diese TCR- $\alpha$-Kette tritt nur in Kombination mit einem begrenzten Repertoire von $\beta$-Ketten auf $\left(\mathrm{V} \beta_{2}\right.$, $\mathrm{V} \beta 7$, V $\beta 8.2$ bei der Maus bzw. $\mathrm{V} \beta 11$ beim Menschen).

Im Gegensatz zu konventionellen TZellen, welche Peptidantigene auf MHCI- oder -II-Molekülen erkennen, erkennt die iNKT-Zelle Lipidantigene, die auf CD1-Molekülen präsentiert werden. CD1Moleküle, die mit MHC-Molekülen evolutionär verwandt sind, sind nichtpolymorphe transmembrane Proteine, die mit $\beta_{2}$-Microglobulin assoziieren und unterschiedliche Lipide an die T-Zellen präsentieren $[1,5]$. Während es beim Menschen 5 Isoformen der CD1-Moleküle gibt (CD1 a-e), besitzt die Maus nur die - auch in allen Spezies am weitesten konservierte - Isoform CDid [13].

Obwohl iNKT-Zellen weniger als $1 \%$ aller T-Zellen ausmachen, spielen sie of- 


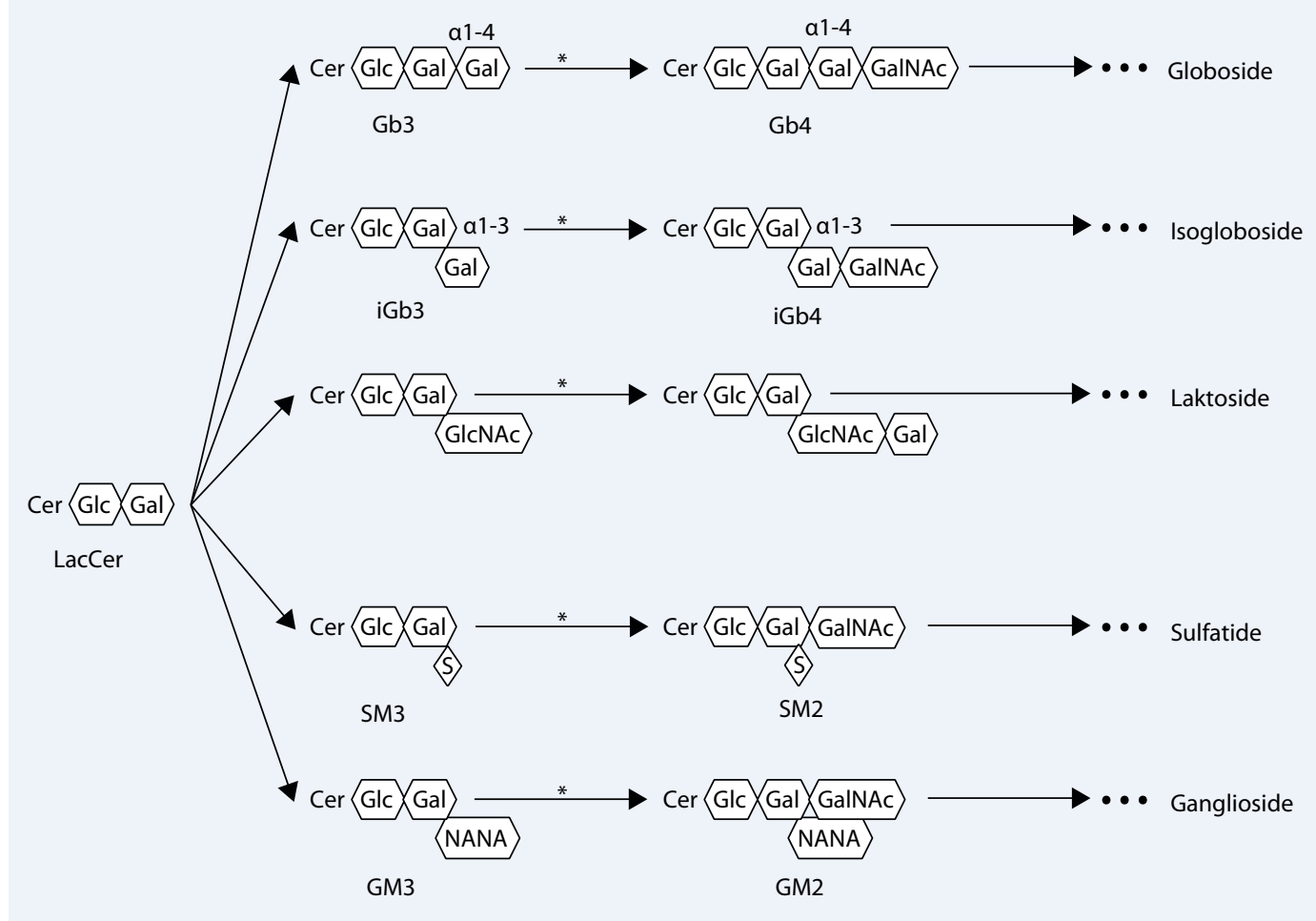

Abb. $1 \Delta$ Vereinfachtes Syntheseschema der Glykosphingolipide (GSL), deren Mehrzahl sich vom Laktosylceramid (LacCer) herleitet. Durch die Addition eines dritten Zuckerrestes bzw. eines Sulfats entstehen die unterschiedlichen Gruppen der GSL. Sowohl das Globotrihexosylceramid (Gb3) als auch das Isoglobotrihexosylceramid ( $i G b 3$ ) haben an der 3. Stelle eine Galaktose, unterscheiden sich jedoch in der glykosidischen Bindung, die im Falle vom Gb3 a1-4 bzw. im Falle vom iGb3 a1-3 ist. Der Abbau dieser Substanzen erfolgt in umgekehrter Richtung und bedarf an der markierten Stelle $\left.{ }^{*}\right)$ der $\beta$-Einheit $\operatorname{der} \beta$-Hexosaminidasen, welche bei der Sandhoff-Speicherkrankheit defekt ist. (Cer Ceramid, Gal Galaktose, GalNAc N-Acetylgalakosamin, Glc Glukose, GICNAC N-Acetylglukosamin, NANA N-Acetylneuraminsäure, S Sulfat)
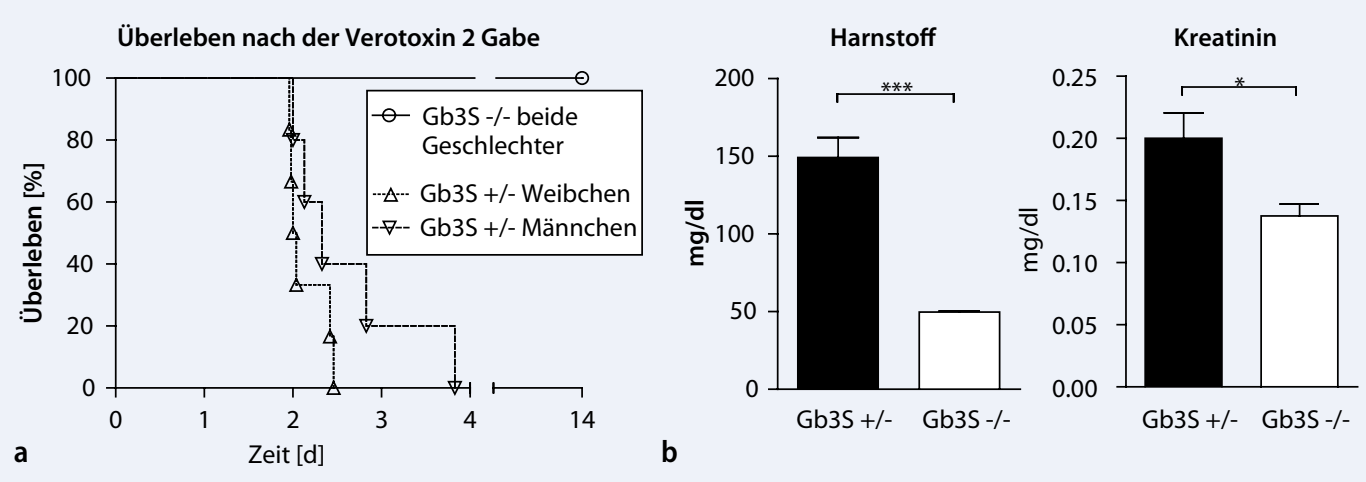

Abb. $2 \Delta$ Gb3S-Defizienz schützt vor der Verotoxintoxizität. Adulten Gb3S-defizienten Mäusen (Gb3S $\left.\mathrm{S}^{-/}\right)$und heterozygoten Kontrollen $\left(\mathrm{Gb3S}^{+-}\right)$wurde intraperitoneal Verotoxin injiziert. a Der spontane Verlauf der Erkrankung wurde abgewartet. Dieser zeigte, dass im Gegensatz zu den Kontrolltieren, die zwischen dem 2. und 4. Tag starben, Gb3S-defiziente Mäuse vor Verotoxintoxizität vollständig geschützt sind ( $p<0,0001$ im Mantel-Cox-Test; $n=11$ pro Gruppe). b 36 Stunden nach der Injektion wurden Plasmakonzentrationen von Harnstoff und Kreatinin bestimmt. Während beide Werte bei Gb3S-defizienten Mäusen im Normbereich lagen, kam es bei den Kontrolltieren zu einem signifikanten $\left({ }^{*}, p<0,05 ;{ }^{* * *}, p<0,001\right)$ Anstieg (Durchschnitt \pm SEM, $n=8$ pro Gruppe)

fensichtlich eine wichtige Rolle in der Abwehr gegen Bakterien (z. B. Mykoplasmen und Spirocheten), Pilze (z. B. Kryptokokken) und Tumoren sowie bei der peripheren Toleranz $[3,17]$.
In Analogie zu konventionellen T-Zellen, die im Thymus durch Peptidantigene auf MHC-I- oder-II-Molekülen positiv selektioniert werden, unterliegen die iNKTZellen einer positiven Selektion durch endogene Lipidantigene auf CDid-Mole- külen [18]. Die Identität dieses (bzw. dieser) endogenen Liganden bleibt jedoch weiterhin unbekannt. Es könnte sich allerdings um ein GSL handeln, da gezeigt wurde, dass Glukosylceramid-defiziente Zelllinien iNKT-Zell-Hybridome nicht 
stimulieren können [16]. Ferner wurde gezeigt, dass Mäuse mit mutierter $\beta$-Einheit der $\beta$-Hexosaminidasen $\left(\mathrm{Hexb}^{-/}\right.$, ein Mausmodel der Sandhoff-Speicherkrankheit, - Abb. 1) eine deutlich reduzierte Anzahl der iNKT-Zellen aufweisen [20]. Daraus wurde geschlossen, dass die bei der Sandhoff-Speicherkrankheit defizienten lysosomalen Degradationsenzyme für die Generierung des gesuchten Lipidliganden verantwortlich sind. Unter den bekannten Degradationsprodukten dieser Enzyme zeigte nur iGb3 eine in vitro stimulierende Kapazität gegenüber den iNKT-Zellen $[20,19]$. Aufgrund dieses indirekten Beweises wurde iGb3 als der endogene Ligand, der für die positive Selektion von iNKT-Zellen verantwortlich ist, postuliert.

Wir haben einen direkten genetischen Ansatz gewählt, um zu testen, ob iGb3 ein physiologisch relevanter Ligand für die positive Selektion der iNKT-Zellen ist. Deshalb wurde eine iGb3-Synthase-defiziente Maus $\left(\mathrm{iGb}_{3} \mathrm{~S}^{-/-}\right.$) generiert und die Entwicklung und Funktion der iNKT-Zellen in diesen Mäusen analysiert.

\section{Methoden}

\section{Herstellung Gb3-Synthase- defizienter Mäuse}

Eine Gb3-Synthase-defiziente Maus wurde mit Hilfe homologer Rekombination in Mausstammzellen konstruiert, indem das letzte Exon des Gb3-Synthase-Gens, das auch für die katalytische Domäne des Enzyms kodiert, durch eine NeomycinKassette ersetzt wurde. Adulte $\mathrm{Gb}_{3} \mathrm{~S}^{+/-}$und $\mathrm{Gb}_{3} \mathrm{~S}^{-/-}$-Mäuse beider Geschlechter wurden mit $0,1 \mu \mathrm{g}$ Verotoxin 2 (Sigma, Schnelldorf, Deutschland) intraperitoneal gespritzt. In einer Gruppe wurden alle Mäuse nach 36 Stunden getötet, um Blutund Organproben zu gewinnen. In einer anderen Gruppe wurde der spontane Verlauf der Erkrankung abgewartet, um eine Überlebensanalyse durchzuführen.

\section{Herstellung und Analyse iGb3- Synthase-defizienter Mäuse}

Eine iGb3-Synthase-defiziente Maus wurde mit Hilfe homologer Rekombination in Mausstammzellen konstruiert, indem das

Pathologe 2008 · [Suppl 2] 29:297-302 DOI 10.1007/s00292-008-1040-0

(c) Springer Medizin Verlag 2008

S. Porubsky · B. Luckow · M. Bonrouhi · A. Speak · V. Cerundolo · F. Platt · H.-J. Gröne Glykosphingolipide Gb3 und iGb3. In-vivo-Rolle im hämolytischurämischen Syndrom und bei der Funktion der iNKT-Zellen

\section{Zusammenfassung}

Die Glykosphingolipide Globotrihexosylceramid (Gb3, CD77) und Isoglobotrihexosylceramid (iGb3) sind Isomere, welche sich nur in einer glykosidischen Bindung unterscheiden. Beide wurden in unterschiedlichen Prozessen der angeborenen und erworbenen Immunität beschrieben.

Ziele. 1. Da Gb3 als der zelluläre Rezeptor für das durch Shigellen und manche $E$. coli-Stämme produzierte Verotoxin (VT) postuliert wurde, wollten wir in Gb3-defizienten Mäusen in vivo testen, ob Gb3 alleine für die Entstehung des durch VT hervorgerufenen hämolytisch-urämischen Syndroms verantwortlich ist. 2. Da iGb3 als der endogene Ligand für die positive Selektion von invarianten "natural-killer-" T-Zellen (iNKT) im Thymus vorgeschlagen wurde, wollten wir diese Hypothese in iGb3-defizienten Mäusen in vivo überprüfen.

Methoden. Konstruktion Gb3- und iGb3defizienter Mäuse. VT-Injektion in Gb3-defi- ziente Mäuse. Analyse der Entwicklung und Funktion der iNKT-Zellen mit Hilfe der Durchflusszytometrie. Verabreichung von a-Galaktosylceramid in iGb3-defiziente Mäuse.

Ergebnisse. 1.Gb3-defiziente Mäuse zeigten sich als unempfindlich gegenüber sonst letalen Dosen von VT. 2. iGb3-defiziente Mäuse zeigten normale iNKT-Zellzahlen. Die Funktion von iNKT-Zellen war in iGb3-defizienten Mäusen ebenfalls unbeeinträchtigt.

Schlussfolgerungen. Gb3 ist der zelluläre Rezeptor, der für die Vermittlung der VT-Toxizität und somit für die Entstehung des hämolytisch-urämischen Syndroms verantwortlich ist. Im Gegensatz zu vorherigen Hinweisen, kann iGb3 kein endogener Ligand sein, der für die positive Selektion von iNKT-Zellen verantwortlich wäre.

Schlüsselwörter iNKT · iGb3 · Gb3 · HUS-TTP

\section{Glycosphingolipids $\mathrm{Gb} 3$ and iGb3. In vivo roles in hemolytic-uremic syndrome and iNKT cell function}

\section{Abstract}

The glycosphingolipids globotrihexosylceramide (Gb3, CD77) and isoglobotrihexosylceramide (iGb3) are isomers differing only in one glycosidic bond and have been implicated in several processes of the innate and adaptive immune system.

Aims. 1) To verify the function of $\mathrm{Gb} 3$ in the pathogenesis of hemolytic-uremic syndrome as the cellular receptor responsible for cytotoxicity caused by verotoxin (VT) elaborated by Shigella and certain strains of E.coli. 2) To investigate in vivo the previously implicated function of iGb3 as the endogenous lipid ligand responsible for positive selection of invariant natural killer T-cells (iNKT), which have an essential regulatory function in infection, tumor rejection and tolerance.

Methods. Generation of mice deficient in Gb3 and iGb3 synthesizing enzymes and VT injection into Gb3-deficient mice. Analysis of
iNKT cell development and function by flow cytometry and by administration of the exogenous agonist alpha-galactosylceramide in iGb3-deficient mice.

Results. For 1) Gb3-deficient mice were insensitive to otherwise lethal doses of VT, and 2) iGb3-deficient mice showed normal numbers of iNKT cells. Furthermore the function of iNKT cells evolving in iGb3-deficient mice was unaffected.

Conclusions. 1) Gb3 is the cellular receptor mediating verotoxin cytotoxicity in haemolytic-uremic syndrome. 2) In contrast to previous indirect implications, iGb3 cannot be regarded as an endogenous ligand responsible for the positive selection of iNKT cells.

Keywords

iNKT · iGb3 · Gb3 · HUS-TTP 


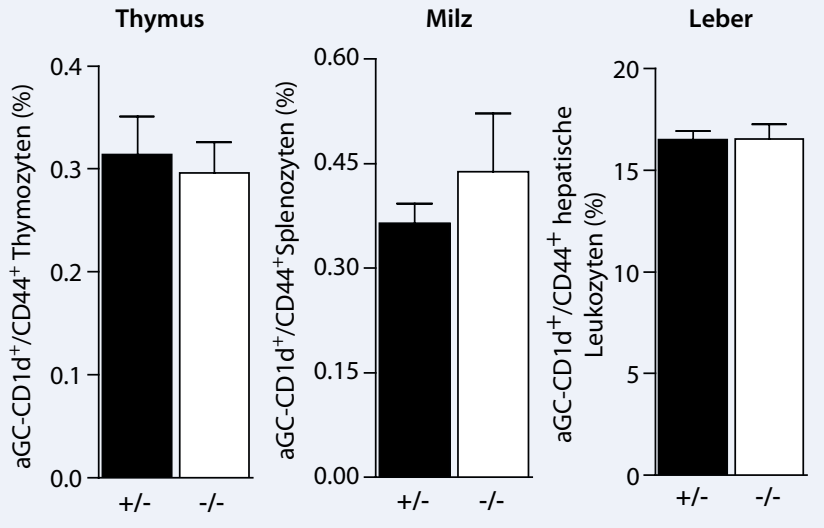

Abb. $3 \Delta$ iGb3S-defiziente Mäuse zeigen unbeeinträchtigte iNKT-Zell-Zahlen. iNKT-Zellen wurden in Organhomogenaten des Thymus, der Milz und der Leber mit Hilfe von Anti-CD44-Antikörper- und aGalCer-beladener CD1d-Tetramere in der Durchflusszytometrie dargestellt. Die Balken geben die Frequenz von iNKT-Zellen im jeweiligen Organ im Lymphozytenfenster an (Durchschnitt \pm SEM, $n=5$ pro Gruppe). Es ergab sich kein statistisch signifikanter Unterschied zwischen den iGb3S-defizienten Mäusen $\left(^{(-)}\right.$und den heterozygoten Kontrollen $\left(^{(+-}\right)$

5. Exon des iGb3-Synthase-Gens, das auch für die katalytische Domäne des Enzyms kodiert, durch eine Neomycin-Kassette ersetzt wurde [14].

Analysen der GSL-Zusammensetzung wurden mittels „,high performance liquid chromatography" (HPLC) durchgeführt [14]. Einzelzellsuspensionen von Thymi und Milzen wurden durch enzymatischen Gewebsverdau mittels Collagenase IA und DNAseI vorbereitet. Die Präparation von Leukozyten aus der Leber erfolgte mit Hilfe eines Percoll-Gradienten [7]. PE-markierte CD1d-Tetramere (Proimmune, Oxford, UK) wurden mit aGalCer (Axxora, Lörrach, Deutschland) beladen [10]. Etwa $10^{6}$ Zellen wurden dann mit 1 pmol entweder $\alpha$ GalCer-beladenen CD1d-Tetramers oder unbeladenen CD1d-Tetramers gefärbt. Färbungen mit anderen Antikörpern wurden durchgeführt, wie beschrieben in [14].

Für die Analyse der Zytokinprofile wurden adulte weibliche $\mathrm{iGb}_{3} \mathrm{~S}^{+/-}$- sowie $\mathrm{iGb}_{3} \mathrm{~S}^{-1-}$-Mäuse intravenös mit $1 \mu \mathrm{g}$ aGalCer (gelöst in $100 \mu \mathrm{l}$ 0,5\% Tween 20 in PBS) gespritzt. Blutproben wurden nach 3 Stunden gesammelt, und die Konzentrationen von IL-6, IL-10, IL-12p7o, IFN$\gamma$, TNF- $\alpha, \mathrm{Ccl}_{2} / \mathrm{MCP}-1$ und IL-4 wurden mit Hilfe der „cytometric bead array-“ (CBA-)Technologie und FACSCalibur ${ }^{\text {Tm }}$ (BD, Heidelberg, Deutschland) gemessen und mit Hilfe der FCAP-Software (Soft Flow, Pécs, Ungarn) analysiert.

\section{Ergebnisse}

\section{Gb3-defiziente Mäuse sind unempfindlich gegenüber letalen Dosen vom Verotoxin 2}

Um die Rolle von Gb3 als Rezeptor für Verotoxin 2 zu überprüfen, konstruierten wir $\mathrm{Gb}_{3} \mathrm{~S}$-defiziente Mäuse $\left(\mathrm{Gb}_{3} \mathrm{~S}^{-/-}\right)$. Chemisch zeigte sich kein Unterschied zwischen Wildtyp-Mäusen $\left(\mathrm{Gb}_{3} \mathrm{~S}^{+/+}\right)$ und Heterozygoten $\left(\mathrm{Gb}_{3} \mathrm{~S}^{+/-}\right)$, weswegen die Letzteren weiter als Kontrolltiere für die $\mathrm{Gb}_{3} \mathrm{~S}^{-1-}$-Mäuse verwendet wurden. Bei nativen $\mathrm{Gb}_{3} \mathrm{~S}^{-/-}$-Mäusen waren keine histomorphologischen Abweichungen bei der Untersuchung vom Gehirn, Auge, Thymus, Herz, Lunge, Leber, Milz, Pankreas, Gastrointestinaltrakt, Niere, Nebenniere, Haut, Knochenmark und Geschlechtsorganen feststellbar. Das Fehlen von Gb3 war ebenfalls weder mit Auffälligkeiten im Verhalten noch gestörter Reproduktion verbunden.

Nach der Verabreichung von Verotoxin 2 verstarben alle $\mathrm{Gb}_{3} \mathrm{~S}^{+/-}$-Mäuse zwischen Tag 2 und 4 (• Abb. 2 a). Die männlichen $\mathrm{Gb}_{3} \mathrm{~S}^{+/-}$-Mäuse zeigten dabei ein etwas verzögertes Versterben, das jedoch im Vergleich zum Verlauf bei den Weibchen nicht statistisch signifikant unterschiedlich war. Im Gegensatz dazu überlebten die Gb3-defizienten Mäuse beider Geschlechter diese Zeitspanne und zeigten auch weiterhin keine Auffälligkeiten während der ganzen Beob- achtungszeit von 14 Tagen ( $\bullet$ Abb. 2 a). Während die $\mathrm{Gb}_{3} \mathrm{~S}^{+/-}$-Mäuse 36 Stunden nach der Verotoxingabe laborchemische Korrelate der Urämie in Form von ansteigenden Retentionswerten (Harnstoff und Kreatinin) aufwiesen, blieben beide Parameter bei den $\mathrm{Gb}_{3} \mathrm{~S}^{-/-}$-Mäusen im Normbereich (• Abb. 2 b).

\section{iGb3-defiziente Mäuse zeigen eine normale Zahl und Funktion von iNKT-Zellen}

Die homologe Rekombination am iGb3SLokus wurde durch „Southern blot“ überprüft und die Absenz des iGb3 sowie der ganzen Isogloboserie der GSL mit Hilfe der HPLC belegt [14]. Es zeigte sich kein Unterschied zwischen Wildtyp-Mäusen $\left(\mathrm{iGb}_{3} \mathrm{~S}^{+/+}\right)$und heterozygoten $\left(\mathrm{iGb}_{3} \mathrm{~S}^{+/-}\right.$ ), weswegen die Letzteren weiter als Kontrolltiere verwendet wurden. Um die Rolle von iGb3 als endogener Ligand, der für die positive Selektion von iNKT-Zellen impliziert wurde, zu überprüfen, untersuchten wir mit Hilfe aGalCer-beladener CD1d-Tetramere die Frequenz der iNKTZellen im Thymus, in der Milz und Leber der $\mathrm{Gb}_{3} \mathrm{~S}^{-/-}$-Mäuse im Vergleich zu den $\mathrm{iGb}_{3} \mathrm{~S}^{+/-}$-Mäusen. iNKT-Zellen kamen in diesen Organen bei den $\mathrm{iGb}_{3} \mathrm{~S}^{-1-}$-Mäusen mit einer nicht verminderten Frequenz vor (- Abb. 3). Ein statistisch signifikanter Unterschied im Vergleich zu den $\mathrm{iGb}_{3} \mathrm{~S}^{+/-}$-Kontrollen konnte nicht festgestellt werden.

Nach der Stimulation mit aGalCer sezernieren die iNKT-Zellen eine Reihe von Zytokinen und üben einen aktivierenden Effekt auf dendritische Zellen aus. Um die In-vivo-Funktion der iNKT-Zellen der $\mathrm{iGb}_{3} \mathrm{~S}^{+/-}$- und $\mathrm{iGb}_{3} \mathrm{~S}^{-/-}$-Mäuse $\mathrm{zu}$ vergleichen, wurde den Mäusen aGalCer verabreicht. Auch hier zeigte sich kein Unterschied zwischen den iNKT-Zellen der iGb3-defizienten Mäuse und der Kontrollen (• Abb. 4 a). Hiermit übereinstimmend ergab sich in diesem Versuch kein Unterschied in der Hochregulation des oberflächlichen CD69-Moleküls bzw. der Zytokine IFN- $\gamma$ und IL-4, welche mit Hilfe der Durchflusszytometrie bestimmt wurden ( Abb. 4 b). Ferner konnte in dieser Versuchsanordnung auch eine unterschiedliche Reifung der dendritischen 


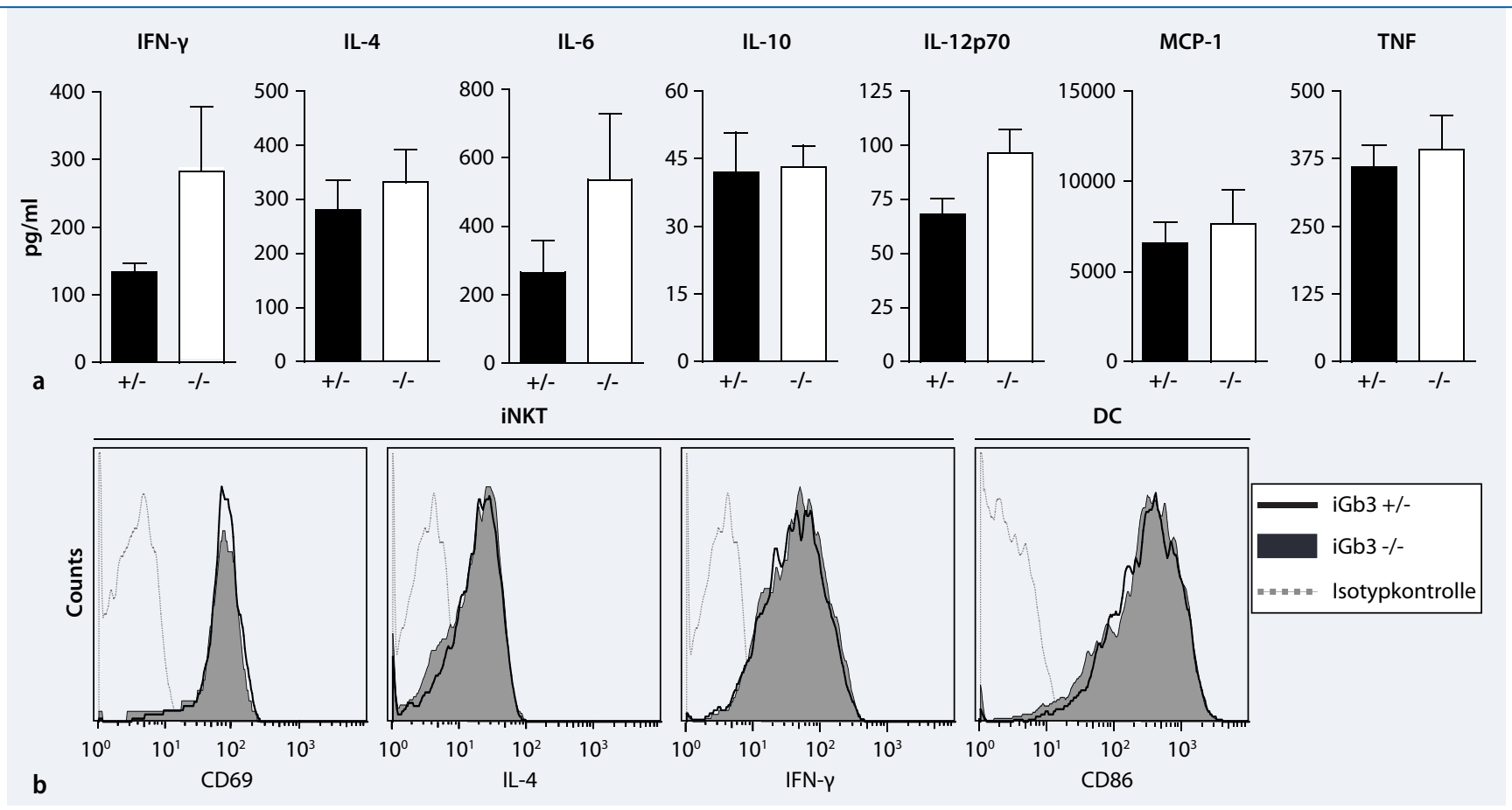

Abb. $4 \Delta$ iNKT-Zellen iGb3S-defizienter Mäuse zeigen eine normale In-vivo-Funktion. iGb3-defizienten Mäusen $\left(^{-/-}\right)$und heterozygoten Kontrollen $\left({ }^{+/}\right)$wurde aGalCer intravenös injiziert. a 3 Stunden nach der Injektion wurde die Konzentration der angezeigten Zytokine im Serum gemessen. iGb3S-/-Mäuse unterschieden sich nicht von den Kontrollen (Durchschnitt \pm SEM, $\mathrm{n}=5$ pro Gruppe). b 2 Stunden nach der Injektion wurden hepatische iNKT-Zellen mittels Durchflusszytometrie auf ihre Expression von CD69, IL-4 und IFN- $\gamma$ untersucht. Weiterhin wurde 12 Stunden nach der Injektion die Expression von CD86 auf dendritischen Zellen (DC) der Milz bestimmt. Gezeigt werden repräsentative Proben für beide Genotypen und die jeweilige Isotypkontrolle. In keinem der Fälle fand sich ein Unterschied zwischen iGb3S $S^{-1-}$ - und iGb3S $\mathrm{S}^{+/-}$-Mäusen

Zellen bei den $\mathrm{iGb}_{3} \mathrm{~S}^{-/-}$-Mäusen ausgeschlossen werden (• Abb. 4 b).

\section{Diskussion}

Die Glykosphingolipide Gb3 und iGb3, welche identische Zuckerreste, jedoch eine unterschiedliche glykosidische Bindung zwischen den letzten 2 Galaktosen aufweisen, wurden in unterschiedlichen (patho-)physiologischen Prozessen involviert.

Mehrere In-vitro-Versuche erbrachten Hinweise, dass $\mathrm{Gb}_{3}$ (CD77) als ein Rezeptor dient, welcher für die intrazelluläre Aufnahme von Verotoxin verantwortlich ist $[4,9]$. Sollte Gb3 (bzw. die von der $\mathrm{Gb}_{3} \mathrm{~S}$ abhängigen GSL) das essenzielle Molekül an der Zelloberfläche sein, das die Bindung und die darauf folgende Endozytose von Verotoxin vermittelt, würde man in den $\mathrm{Gb}_{3} \mathrm{~S}^{-/}$-Mäusen keine Toxizitätserscheinungen erwarten. Dies konnte hier in einem In-vivo-Versuch bestätigt werden. Die Anwesenheit von $\mathrm{Gb}_{3}$ bzw. anderer GSL der Globoserie ist somit eine Conditio sine qua non für die Entstehung des urämischen Syndroms nach Verotoxinexposition. Ohne $\mathrm{Gb}_{3}$ führen selbst letale Dosen von Verotoxin $2 \mathrm{zu}$ keinerlei Organschäden.

Ähnliche Ergebnisse wurden von einer anderen, von uns unabhängig konstruierten, $\mathrm{Gb}_{3} \mathrm{~S}$-defizienten Maus veröffentlicht [12]. Es bleibt jedoch weiterhin fraglich, welche Zellen bzw. Struktur(en) in der Entstehung des hämolytisch-urämischen Syndroms eine führende Rolle haben und welche weiteren Organschäden erst die Folge hiervon sind. Die Verotoxintoxizität kommt erst nach der Depletion vorbestehender Proteine durch endogene Umsatzmechanismen zum Vorschein. Deshalb lässt sich vermuten, dass die Zelle, welche neben ausreichender Gb3-Expression auch einen hohen Proteinumsatz aufweist, diejenige Struktur im Körper sein könnte, die in der Pathogenese des hämolytisch-urämischen Syndroms führend ist. Dies ist der Gegenstand unserer weiteren Untersuchungen.

Aufgrund früherer Untersuchungen in $\mathrm{Hexb}^{-/-}$Mäusen, die in Lysosomen u. a. - iGb4 zu iGb3 nicht spalten können
(• Abb. 1) und die keine iNKT-Zellen aufweisen, wurde iGb3 als der endogene Ligand vorgeschlagen, der für die positive Selektion dieser Zellen im Thymus verantwortlich ist [20]. Obwohl $\mathrm{iGb}_{3}$ in vitro eine stimulatorische Kapazität gegenüber iNKT-Zellen besitzt $[20,19]$, gibt es keinen direkten Beweis dafür, das iGb3 in vivo die Rolle des endogenen Liganden erfüllt. Diese Hypothese wollten wir direkt überprüfen und konstruierten deshalb iGb 3 S-defiziente Mäuse. $\mathrm{iGb}_{3} \mathrm{~S}^{-/}$-Mäuse hatten keine nachweisbaren GSL der Isogloboserie (iGb3, iGb4, iGb5; [14]). a1,3Galaktosyltransferase, die ähnlich wie $\mathrm{iGb}_{3} \mathrm{~S}$ für die Synthese von Gala1-3GalEpitopen verantwortlich ist, konnte die Aktivität der ausgeschalteten $\mathrm{iGb}_{3} \mathrm{~S}$ nicht ersetzen. Dies entspricht den früheren Beobachtungen über die Substratspezifizität dieser 2 Enzyme: a1,3-Galaktosyltransferase ist spezifisch für das auf Proteinen befindliche N-Acetyllactosamin, während $\mathrm{iGb}_{3} \mathrm{~S}$ spezifisch für GSL ist [11].

Wir fanden, dass sich die Anzahl und Funktion der iNKT-Zellen in $\mathrm{iGb}_{3} \mathrm{~S}^{-/-}$ Mäusen nicht von den $\mathrm{iGb}_{3} \mathrm{~S}^{+/-}$-Kontrol- 
len unterscheidet. Dieser Befund spricht stark gegen die frühere Annahme, dass iGb3 die thymische Selektion der iNKTZellen vermittelt. Ferner zeigte sich inzwischen, dass der Verlust der iNKT-Zellen nicht spezifisch für Defekte im Metabolismus des Isoglogoserie ist (z. B. $\mathrm{Hexb}^{-/-}$), sondern dass man eine Reduktion der iNKT-Zellzahl auch in anderen Speichermodellen beobachten kann, welche mit dem Metabolismus der Isogloboside nicht direkt interferieren [2]. Aus diesem Grund ist die Absenz der iNKT-Zellen in diesen Speichermodellen vielmehr die Konsequenz der Lipidspeicherung als der Abwesenheit von iGb3. In Zusammenschau aller Ergebnisse zeigt sich somit, dass iGb3 für die positive Selektion und periphere Funktion der iNKT-Zellen nicht verantwortlich ist und dass ein anderer Ligand bzw. andere Liganden gesucht werden müssen.

\section{Korrespondenzadresse \\ Dr. S. Porubsky}

Zelluläre und molekulare Pathologie Deutsches Krebsforschungszentrum Im Neuenheimer Feld 280, 69120 Heidelberg s.porubsky@dkfz.de

Interessenkonflikt. Der korrespondierende Autor gibt an, dass kein Interessenkonflikt besteht.

\section{Literatur}

1. Brigl M, Brenner MB (2004) CD1: antigen presentation and T cell function. Annu Rev Immunol 22: 817-890

2. Gadola SD, Silk JD, Jeans A et al. (2006) Impaired selection of invariant natural killer T cells in diverse mouse models of glycosphingolipid lysosomal storage diseases. J Exp Med 203: 2293-2303

3. Hansen DS, Schofield L (2004) Regulation of immunity and pathogenesis in infectious diseases by CD1d-restricted NKT cells. Int J Parasitol 34: 15-25

4. Jacewicz M, Clausen H, Nudelman E et al. (1986) Pathogenesis of shigella diarrhea. XI. Isolation of a shigella toxin-binding glycolipid from rabbit jejunum and hela cells and its identification as globotriaosylceramide. J Exp Med 163: 1391-1404

5. Kasahara M, Nakaya J, Satta Y, Takahata N (1997) Chromosomal duplication and the emergence of the adaptive immune system. Trends Genet 13: 90-92

6. Khine AA, Lingwood CA (1994) Capping and receptor-mediated endocytosis of cell-bound verotoxin (Shiga-like toxin). 1: Chemical identification of an amino acid in the B subunit necessary for efficient receptor glycolipid binding and cellular internalization. J Cell Physiol 161: 319-332
7. Korten S, Anderson RJ, Hannan CM et al. (2005) Invariant Valpha14 chain NKT cells promote plasmodium berghei circumsporozoite protein-specific gamma interferon- and tumor necrosis factor alpha-producing CD8+ T cells in the liver after poxvirus vaccination of mice. Infect Immun 73: 849-858

8. Kronenberg M, Gapin L (2002) The unconventional lifestyle of NKT cells. Nat Rev Immunol 2: 557-568

9. Lingwood CA, Law H, Richardson S et al. (1987) Glycolipid binding of purified and recombinant escherichia coli produced verotoxin in vitro. J Biol Chem 262: 8834-8839

10. Matsuda JL, Naidenko OV, Gapin L et al. (2000) Tracking the response of natural killer T cells to a glycolipid antigen using CD1d tetramers. J Exp Med 192: 741-754

11. Milland J, Christiansen D, Lazarus BD et al. (2006) The molecular basis for galalpha(1,3) gal expression in animals with a deletion of the alpha1,3galact osyltransferase gene. J Immunol 176: 2448-2454

12. Okuda T, Tokuda N, Numata S et al. (2006) Targeted disruption of Gb3/CD77 synthase gene resulted in the complete deletion of globo-series glycosphingolipids and loss of sensitivity to verotoxins. J Biol Chem 281: 10230-10235

13. Porcelli SA, Modlin RL (1999) The CD1 system: antigen-presenting molecules for $T$ cell recognition of lipids and glycolipids. Annu Rev Immunol 17: 297329

14. Porubsky S, Speak AO, Luckow B et al. (2007) Normal development and function of invariant natural killer T cells in mice with isoglobotrihexosylceramide (iGb3) deficiency. Proc Natl Acad Sci U S A 104: 5977-5982

15. Sandvig K, Garred O, Prydz K et al. (1992) Retrograde transport of endocytosed shiga toxin to the endoplasmic reticulum. Nature 358: 510-512

16. Stanic AK, De Silva AD, Park JJ et al. (2003) Defective presentation of the CD1d1-restricted natural Va14Ja18 NKT lymphocyte antigen caused by beta-D-glucosylceramide synthase deficiency. Proc Natl Acad Sci U S A 100: 1849-1854

17. Taniguchi M, Harada M, Kojo S et al. (2003) The regulatory role of Valpha14 NKT cells in innate and acquired immune response. Annu Rev Immunol 21: 483-513

18. Wei DG, Curran SA, Savage PB et al. (2006) Mechanisms imposing the Vbeta bias of Valpha14 natural killer T cells and consequences for microbial glycolipid recognition. J Exp Med 203: 1197-1207

19. Xia C, Yao Q, Schumann J et al. (2006) Synthesis and biological evaluation of alpha-galactosylceramide (KRN7000) and isoglobotrihexosylceramide (iGb3). Bioorg Med Chem Lett 16: 2195-2199

20. Zhou D, Mattner J, Cantu C 3rd et al. (2004) Lysosomal glycosphingolipid recognition by NKT cells. Science 306: 1786-1789 